\title{
Long term management of patients with cryopyrin-associated periodic syndromes (CAPS): focus on rilonacept (IL-I Trap)
}

\author{
Leigh D Church' \\ Sinisa Savic ${ }^{2}$ \\ Michael F McDermott ${ }^{2}$ \\ 'Department of Rheumatology, \\ Division of Immunity, Infection \\ and Inflammation, Institute \\ for Biomedical Research, The University \\ of Birmingham, Birmingham, UK; \\ ${ }^{2}$ Section of Musculoskeletal Disease, \\ Leeds Institute of Molecular Medicine, \\ Wellcome Trust Brenner Building, \\ University of Leeds, Leeds, UK
}

\begin{abstract}
Cryopyrin-associated periodic syndromes (CAPS) are a group of inherited inflammatory disorders consisting of familial cold-induced autoinflammatory syndrome (FCAS), Muckle-Wells syndrome (MWS), and neonatal-onset multisystem inflammatory disease (NOMID; also known as chronic infantile neurologic, cutaneous, articular [CINCA] syndrome). These rare disorders are associated with heterozygous mutations in the NLRP3 (CIAS1) gene, which encodes the protein NALP3 or cryopyrin, and inflammation driven by excessive production of the cytokine interleukin$1 \beta$ (IL-1 $)$. Amyloidosis is a serious complication with $25 \%$ of MWS patients developing amyloidosis, with occasional fatal consequences, whilst up to $20 \%$ of CINCA/NOMID patients die from various complications, before reaching the early adulthood. In some CINCA/NOMID adult survivors amyloidosis can also occur. Prior to the discovery of the CIAS1 gene mutations and the advent of IL-1 targeted therapy, treatment was aimed at suppressing inflammation, with limited success. The selective blockade of IL-1 $\beta$, with anakinra (IL-1 receptor antagonist), not only provided supportive evidence for the role of IL- $1 \beta$ in CAPS, but also demonstrated the efficacy of targeting IL- $1 \beta$ for treatment of these conditions. In February, 2008, 'Orphan Drug' approval from the Food and Drug Administration (FDA) for rilonacept (IL-1 Trap/Arcalyst ${ }^{\mathrm{TM}}$, Regeneron Pharmaceuticals, Inc) was given for the treatment of two CAPS disorders, FCAS and MWS in adults and children 12 years and older, making rilonacept the first therapy approved for the treatment of CAPS.
\end{abstract}

Keywords: cryopyrin-associated periodic syndromes, interleukin-1, therapy, rilonacept

\section{Introduction}

Cryopyrin-associated periodic syndrome (CAPS) is a group of inherited inflammatory disorders consisting of familial cold-induced autoinflammatory syndrome (FCAS; also known as familial cold urticaria [FCU]), Muckle-Wells syndrome (MWS), and neonatal-onset multisystem inflammatory disease (NOMID; also known as chronic infantile neurologic, cutaneous, articular [CINCA] syndrome). These disorders are associated with heterozygous mutations in the NLRP3 (CIAS1) gene, which encodes the protein NALP3 or cryopyrin, and inflammation driven by an excessive production of the inflammatory cytokine interleukin-1 $\beta$ (IL-1 $\beta$ ). CAPS disorders are rare, and until recently there was no adequate therapy for their treatment. The elucidation of the pathway involved in IL-1 signalling and its dysregulation underlying CAPS has enabled the development of IL-1-targeted therapies, such as the IL-1 receptor antagonist, anakinra. The aim of this review is to examine the long-term management of CAPS, focusing on rilonacept (IL-1 Trap/Arcalyst ${ }^{\mathrm{TM}}$; Regeneron Pharmaceuticals, Inc), the recent FDA approved treatment for MWS and FCAS in adults and children 12 years and older.

Correspondence: Michael F McDermott Section of Musculoskeletal Disease, Leeds Institute of Molecular Medicine, Wellcome Trust Brenner Building, University of Leeds, St James's University Hospital, Leeds, LS9 7TF, UK

Email m.mcdermott@leeds.ac.uk

\section{Clinical and pathological features of CAPS}

The CAPS family of related conditions share a common genetic ancestry, but manifest as separate disease entities with overlapping clinical features, of variable severity. 
Historically these conditions were identified and described independently of one another, with their diagnosis being based solely on their clinical features, prior to discovery of their common genetic origin.

FCAS was first described in 1940 as a rare disorder inherited in an autosomal dominant manner and characterized by intermittent attacks of rash, fever and arthralgias (Kile and Rusk 1940). MWS was named after two physicians, who, in 1962, first described a British family with childhood onset of recurrent episodes of urticarial-like skin rash, fever, chills, malaise and piercing limb pains, with later development of sensorineural hearing loss and amyloidosis (Muckle and Wells 1962).

The first case of what was later to be known as CINCA in Europe and NOMID in North America, was described by Lorber in 1973 (Lorber 1973). He presented an 11-yearold boy with dwarfism; persistently open fontanelle, recurrent subdural effusions, with temporary alternatesided hemiplegia, high-tone deafness, visual defects with pseudopapilloedema, slowing of intellectual development, recurrent acute polyarthritis, erythema marginatum, splenomegaly and iron-resistant hypochromic anemia. Subsequently, a case series of 30 patients from France was used to clinically define the CINCA/NOMID syndrome (Prieur et al 1987).

FCAS, MWS and CINCA/NOMID share a number of features but also have some distinctive clinical manifestations, which together define the disease continuum that is CAPS, with FCAS at the mild end of the disease spectrum, CINCA/NOMID at the severe end and MWS somewhere in the middle.
All three forms usually present in infancy and early childhood, and symptoms may be present at birth in the case of CINCA/NOMID. In keeping with the varying severity of these conditions, the length of attacks also varies accordingly. In the case of FCAS and MWS, where the onset of an attack is usually marked by fevers and chills, the symptoms last up to 24 hours in FCAS, and up to several days, or rarely continuously in MWS. In CINCA/NOMID the periodicity of the attacks is lost and, instead, there is a low-level continuous disease activity, with an occasional disease flare-up (Table 1).

Urticarial-like skin rash is universally present in all CAPS patients. Although it was claimed that exposure to cold typically precipitates attacks of just FCAS, similar observations have also been made for MWS patients (Haas et al 2004). Unlike acquired cold-induced urticaria, where the skin lesions are localized and induced almost immediately following, for example, ice cube provocation testing, in FCAS and MWS, the onset of symptoms is usually delayed for hours following generalized exposure to cold temperatures and the ice cube provocation test is negative (Haas et al 2004; Hoffman et al 2001). Urticaria may be persistent in CINCA/NOMID patients.

There is also a predictable gradation of severity of articular symptoms, which are universal in all CAPS patients, from arthralgia in FCAS, polyarticular arthritis in MWS to severe joint deformities typical of CINCA/ NOMID. The latter has highly characteristic features, such as distinctive radiographic changes of premature patellar and epiphyseal long bone ossification, leading to osseous overgrowth in early life and subsequent severe

Table I Cryopyrin-associated periodic syndromes (CAPS) features

\begin{tabular}{lllll}
\hline CAPS & & FCAS & MWS & CINCA/NOMID \\
\hline Genetics & Inheritance & Autosomal dominant & Autosomal dominant & Majority are de novo \\
& Locus, gene, protein & Iq44, CIASI, cryopyrin & Iq44,CIASI, cryopyrin & Iq44,CIASI, cryopyrin \\
& Onset & Childhood & Childhood & From birth \\
& Duration of attacks & Up to I day & Several days or continuous & Continuous \\
& Cutaneous & Urticarial rash & Urticarial rash & Urticarial rash, neutrophilic eccrine hidradenitis \\
& Musculoskeletal & Arthralgia & Arthritis & Epiphyseal overgrowth and joint deformity \\
& Neurological & Conjuctivits, & Conjunctivitis, deafness & Meningitis, mental retardation, deafness \\
& Morphological & None & None & Short statute, frontal bossing, delayed closure \\
& & & & of fontanelles, saddle nose, clubbing \\
& Amyloid & No & Yes & Yes \\
\hline
\end{tabular}

Abbreviations: CINCA, chronic infantile neurologic cutaneous articular syndrome; FCAS, familial cold-induced autoinflammatory syndrome; MWS, muckle wells syndrome; NOMID, neonatal-onset multisystem inflammatory disease. 
contractures and disability (Kaufman and Lovell 1986; Torbiak et al 1989).

As a part of the triad that involves dermatological, arthropathic and neurological symptoms, which are typical of CAPS, neurological manifestations of the disease are, again, most severe in CINCA/NOMID and almost non-existent in FCAS. Neurological problems that have been encountered in CINCA/NOMID patients include chronic septic meningitis, cerebral atrophy and mental retardation, cerebral ventricular dilatation, pseudopapilloedema and high-frequency hearing loss (Hashkes and Lovell 1997). The latter is also the principal neurological complication found in MWS, but not in FCAS. All three disease entities have frequent ocular involvement, which include conjunctivitis in FCAS, conjunctivitis and episcleritis in MWS (Farasat et al 2008) and uveitis in CINCA/NOMID (Dollfus et al 2000).

There are a number of morphological features that are typical of CINCA/NOMID alone, and include short stature, enlarged head, saddleback nose and short and thick extremities, with clubbing of fingers. No morphological changes have been observed in FCAS and MWS (Neven et al 2008).

Prior to the availability of current treatments, which mainly consist of various anti-interleukin-1 (IL-1) therapeutics, only FCAS had a benign prognosis. Around $25 \%$ of MWS patients developed amyloidosis (Dode et al 2003), with occasional fatal consequences, whilst up to $20 \%$ of CINCA/NOMID patients died from various complications, such as infection, vasculitis and amyloidosis, before reaching adulthood (Hashkes and Lovell 1997). Amyloidosis is also seen in a proportion of surviving CINCA/NOMID patients.

Most of the signs and symptoms that clinically define CAPS are the result of an inappropriate inflammatory response. Earlier studies have consistently shown elevated white cell counts in the peripheral blood, with predominant polymorphonuclear leukocytosis and raised CRP and ESR during the attacks. Furthermore, skin biopsies of the affected areas have shown an inflammatory infiltrate consisting of neutrophils, eosinophils and lymphocytes, in the absence of mast cells and concentrated around vascular walls of dilated capillaries (Farasat, Aksentijevich, and Toro 2008; Haas, Kuster, Zuberbier, and Henz 2004). MRI investigations of the brain have demonstrated leptomeningial and cochlear enhancement, consistent with oedema and inflammation (Neven et al 2008).

\section{Genetics}

Prior to discovery of genetic mutations responsible for CAPS, the cause of the excessive inflammation was largely unknown. The investigations were limited to exclusion of known autoimmune diseases, by demonstrating the absence of the more common autoantibodies, such as anti-nuclear antibodies (ANA) and rheumatoid factor (RF).

Genetic studies using large families with FCAS and MWS led to identification of chromosome 1q44 as a susceptibility locus for these diseases (Cuisset et al 1999; Hoffman et al 2000). Subsequently, FCAS and MWS were shown to be associated with mutations in the NLRP3 (Nucleotide binding oligomerization domain, Leucine rich Repeat and Pyrin domain containing 3)/CIAS1(ColdInduced Autoinflammatory Syndrome 1) gene, which codes for cryopyrin or NALP3 (Aganna et al 2002; Dode et al 2002b; Hoffman et al 2001). Later still missense mutations in CIAS1 were also found in a number of unrelated CINCA syndrome patients (Feldmann et al 2002). To date more then 70 CAPS associated, mainly missense mutations found in exon 3 of CIAS1, have been registered with the INFEVERS database (http://fmf.igh.cnrs.fr/infevers). There is a variable genotype/phenotype correlation. Some of the mutations are exclusively associated with one of the syndromes, whilst others can produce alternative clinical manifestations. Mutations such as R260W, V198M, V262G can all, for example, result in MWS or FCAS (Dode et al 2002a), whilst on the other hand, Y570, F309SA and F523L are exclusively associated with severe forms of CINCA (Aksentijevich et al 2002). Although CAPS are inherited in an autosomal dominant manner, with a gain of function of the mutated protein, this variability in the clinical manifestations suggests that other modifier genes and environmental factors probably play a significant part in the pathogenesis of these conditions.

In addition to patients who carry recognisable CIASI mutations, there are a number of patients in whom such mutations are not present but have clinically apparent CAPS. The search for mutations in genes that are functionally associated or related to CIAS1, for example ASC (Apoptosis-associated Speck-like protein containing a Caspase recruitment domain), in such patients has so far largely been unsuccessful. A recently discovered mutation in $C A S P 1$, leading to a structurally altered caspase-1 with decreased enzyme activity and a condition provisionally called ICE fever, might suggest an alternative pathway leading to the pro-inflammatory immunopathological profile similar to CAPS. Patients affected by this mutation all suffer from recurrent febrile episodes accompanied by arthralgia, exanthema and elevated systemic markers of inflammation. Although the accepted hypothesis of CAPS pathogenesis (discussed below) postulates the excess of active IL-1 $\beta$ as 


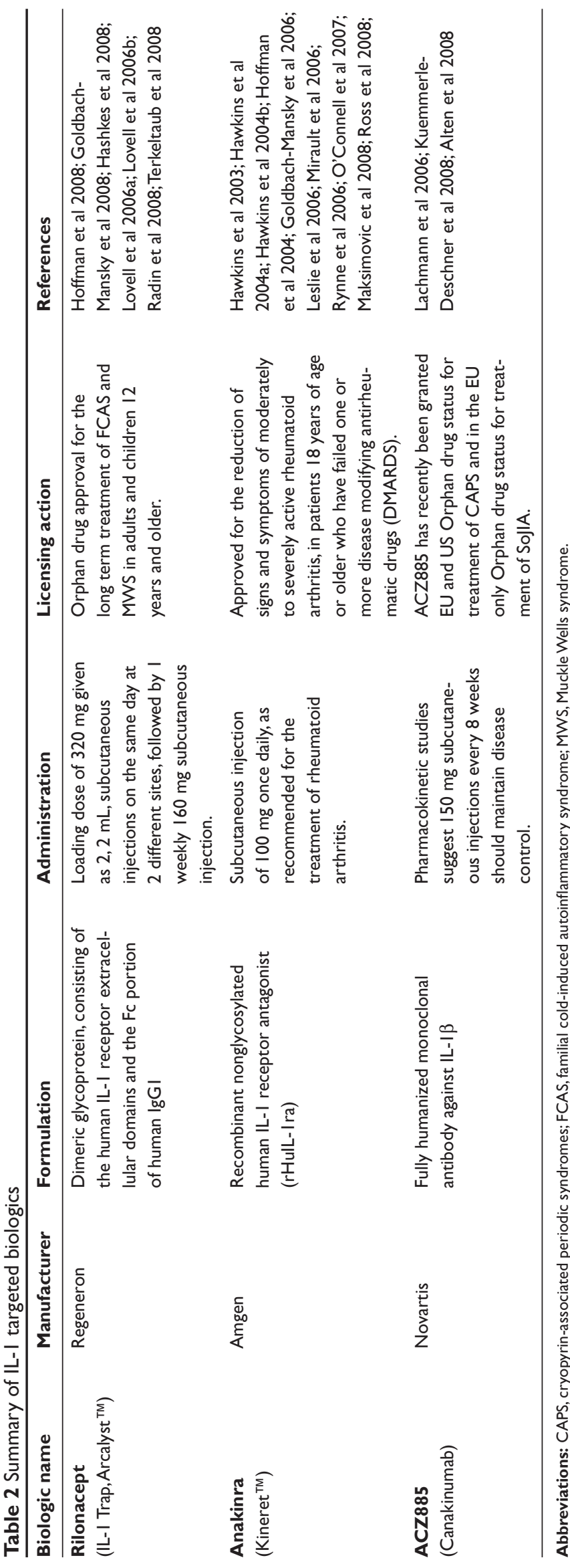


the principal consequence of CIASI mutations leading to the manifestation of the clinical disease, the CASP 1 mutation appears to exert its pro-inflammatory effect by engaging RIP2, resulting in excessive activation of NF- $\kappa B$ and MAPK pathways (Touitou et al 2008).

Another explanation why certain patients have a clinical disease but no obvious genetic mutation has been offered by a Japanese group (Saito 2005, 2008), which has described a CINCA patient with a somatic mosaicism. Careful sequencing and analysis of patient's exon 3 of CIAS1 demonstrated point mutations, 587G $>\mathrm{A}(\mathrm{S} 196 \mathrm{~N})$ and $1709 \mathrm{~A}>\mathrm{G}$ (Y570C). Although the first point mutation was easily identified to be a relatively rare SNP, the second mutation, which is associated with a severe CINCA phenotype, was much harder to identify as the chromatografic intensity of the mutated $\mathrm{G}$ at the position 1709 was considerably lower than that of A at the same position. This was suggestive of somatic mosaicism, whereby only a proportion of somatic cells carried the mutation. Further analysis demonstrated that the mutation occurred with a frequency of 16.7 in whole blood and that it was present in several cell lines. The patient had all the clinical features consistent with CINCA and the analysis of their PBMC showed spontaneous and excessive IL-1 production.

\section{Immunopathology}

Identification of NALP3 as a target protein of CAPS associated mutations has led to a better understanding of immunopathology of CAPS and development of targeted therapeutic interventions.

NALP3 belongs to a large family of proteins, which are collectively known as NLRs [NOD (nucleotide-binding and oligomerization domain) Like Receptors] due to their characteristic tri-domain structure with C-terminal ligand-binding and regulatory LRR (Leucin-Rich Repeat) domain, a central nucleotide-binding and oligomerization NACHT domain and an N-terminal protein-protein interaction domain, which in case of NALP3 is PYD (Ting et al 2006). Like the majority of NLR proteins NALP3 is widely distributed in cells of innate immune cells including macrophages, neutrophils and dendritic cells as well as epithelial cells lining the oral and genital tracts (Kummer et al 2007). This reflects the principal function of this protein, which is to detect danger signals and as a part of multimeric protein complex, known as NALP3 inflammasome, regulate IL-1ß and IL-18 production (Martinon et al 2002). Monosodium urate (Martinon et al 2006), calcium pyrophosphate and silica crystals (Cassel et al 2008), aluminium hydroxide (a vaccine adjuvant) (Eisenbarth et al 2008),
DNA fragments (Muruve et al 2008), ATP and potassium flux (Petrilli et al 2007) have all so far been identified as danger signals recognized by NALP3 in the context of infection and/or tissue damage. Following ligand binding, which is thought but not proven to be via LRR domain, NALP3 undergoes NACHT domain-mediated self-oligomerization. This leads to activation of N-terminal domain and subsequent PYD-PYD homologous protein interaction leading to the recruitment of the ASC adaptor protein, which together with CARDINAL activates caspase-1, which, in turn cleaves pro-IL-1ß into its active form (Church et al 2008; Martinon et al 2002) (Figure 1).

The majority of mutations associated with CAPS are clustered within the highly conserved NACHT domain of NALP3. It has been proposed that these mutations have a gain of function effect, leading to spontaneous oligorimerization of NALP3 subunits around mutated NACHT domains and subsequent caspase-1 activation resulting in an excessive and inappropriate IL-1 $\beta$ release (Agostini et al 2004; McDermott and Aksentijevich 2002). This hypothesis is supported by observations that monocytes from patients with MWS and CINCA/NOMID spontaneously secrete IL-1 $\beta$ (Rosengren et al 2007). Furthermore, therapeutic inhibition of IL-1 $\beta$ action has been shown to be effective in reducing the disease activity and leading to symptomatic improvement in the majority of CAPS patients.

\section{Standard treatment}

Prior to the discovery of mutations of the CIAS1 gene mutation underlying CAPS and the advent of IL-1 targeted therapy, treatment for patients with CAPS was aimed at suppressing inflammation, and have included high-dose corticosteroids, disease-modifying anti-rheumatics drugs (DMARDS) and biologics targeting tumour necrosis factor (TNF), such as etanercept. CAPS patients have unfortunately been relatively refractory to most of these treatments.

\section{IL-I-targeted therapy}

The identification of the CIAS1 mutations and the elucidation of the molecular basis for MWS, FCAS and CINCA/NOMID identified these diseases as part of a disease spectrum associated with the excessive production of IL-1 $\beta$. The selective blockade of IL-1 $\beta$ provided supportive evidence for the role of IL-1 $\beta$ in CAPS, while also demonstrating the effectiveness of targeting IL-1 $\beta$ for the successful treatment of these conditions (Goldbach-Mansky et al 2006; Hawkins et al 2003, 2004a, b; Hoffman et al 2004; Leslie et al 2006; Maksimovic et al 2008; Mirault et al 2006; O’Connell et al 2007; Ross et al 2008; 


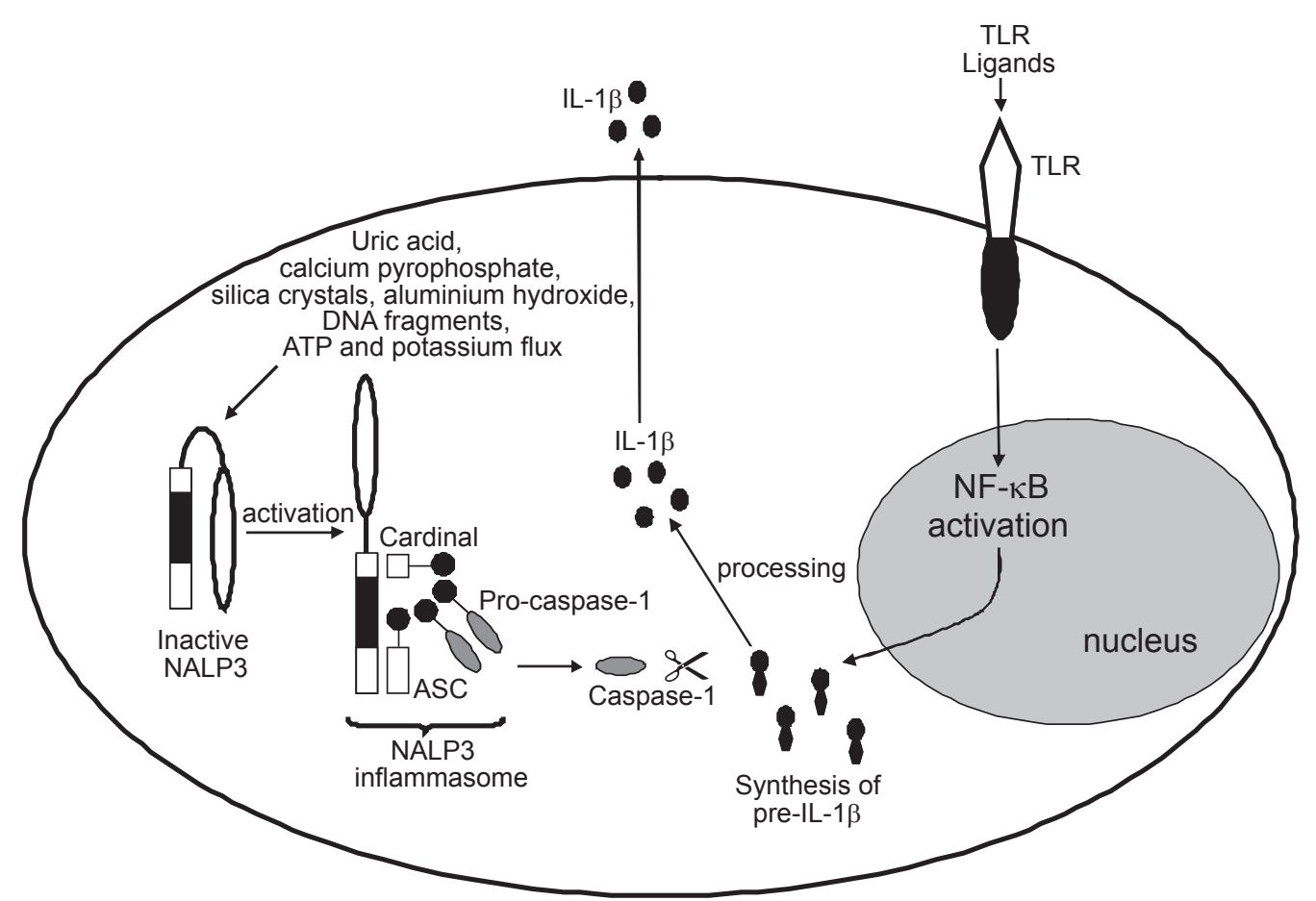

Figure I Activation of the NALP3 inflammasome. Engagement of specific TLRs (Toll-like receptors) leads to activation of NF- $\kappa B$ (nuclear factor $\kappa B$ ) signaling pathways, inducing the expression of pro-IL-I $\beta$. NALP3 is the central component of the NALP3 inflammasome; in its inactive state the LRR (leucine-rich repeat) domain of NALP3 is thought to self-associate, preventing interaction with CARDINAL or ASC (apoptosis-associated speck-like protein containing a CARD). NALP3 activation by agonists, such as ATP, uric acid crystals, bacterial messenger RNA, or aluminium hydroxide, unfolds the NALP3 molecule, enabling the assembly of the inflammasome components CARDINAL,ASC, and pro-caspase-I through homotypic interactions between their respective pyrin (PYD) and CARD (caspase recruitment domain) domains; CARDINAL interacts with the NAD domain of NALP3 through its FIIND (domain with function to find) domain. The oligomerization of inflammasome complexes induces cleavage of pro-caspase-I to its active form, resulting in the generation of active IL-I $\beta$ from its inactive precursor pro-IL-I $\beta$.

Rynne et al 2006). Currently, there are a number of IL-1 inhibitors at various stages of development. These include monoclonal antibodies to IL-1 and 'cytokine traps', such as anakinra (Kineret ${ }^{\mathrm{TM}}$ ), canakinumab (ACZ885), and rilonacept $\left(\right.$ Arcalyst $\left.^{\mathrm{TM}}\right)$.

\section{Anakinra}

Anakinra is a recombinant nonglycosylated human IL-1 receptor antagonist (rHuIL-1 ra) that consists of 153 amino acids with a molecular weight of $17.3 \mathrm{kDa}$, and is identical (except for the addition of one N-terminal methionine) to the naturally occurring form of human IL-1 receptor antagonist (IL-1ra). The use of anakinra by daily subcutaneous injections has been approved for the treatment of rheumatoid arthritis (RA). Despite the modest efficacy of anakinra in RA (Clark et al 2004; Taylor 2003), several studies and case reports following the administration guidelines for RA have now been published, with impressive evidence of its remarkable efficacy in the treatment of MWS, FCAS and CINCA/NOMID (Goldbach-Mansky et al 2006; Hawkins et al 2003, 2004a, b; Hoffman et al 2004; Leslie et al 2006;
Maksimovic et al 2008; Mirault et al 2006; O'Connell et al 2007; Ross et al 2008; Rynne et al 2006). This striking improvement probably reflects the predominance of IL$1 \beta$ in mediating the symptoms associated with CAPS, compared with the complex and multiple inflammatory pathways which underlie the pathogenesis of RA. One of the largest studies, by Goldbach-Mansky and colleagues, recruiting 18 patients with CINCA/NOMID, demonstrated a rapid response to anakinra, with markedly improved clinical and laboratory measures. Rash and conjunctivitis subsided within three days in all cases, whilst diary scores of symptoms were decreased at three months. The ESR, and serum levels of amyloid A and CRP, fell significantly during treatment in all patients (Goldbach-Mansky et al 2006). Interestingly, reports have shown an improvement in hearing in those MWS and CINCA/NOMID patients who received anakinra (Goldbach-Mansky et al 2006; Mirault et al 2006; Rynne et al 2006), while others have found anakinra to stabilize hearing loss (Maksimovic et al 2008). The study by Leslie and colleagues demonstrated that anakinra facilitated the resolution of AA amyloidosis-associated renal dysfunction. 
In that study, serum AA and CRP normalized within 1 week in all patients, while serial SAP scintigraphy revealed regression of amyloid within 8 to 33 months in 3 patients who had AA amyloidosis (Leslie et al 2006). This observation is of particular significance considering that one quarter of patients with MWS will develop AA amyloidosis (Hawkins et al 2004b). The AA amyloidosis usually manifests as impaired renal function, typically leading to renal failure and eventual death within 10 years in the absence of effective therapy. This harsh fact was particularly demonstrated in that study in which 3 of 6 patients in their series developed end-stage renal failure before the advent of anakinra, leading to the death of 2 of them (Leslie et al 2006).

Withdrawal of anakinra treatment led to a very rapid regression of symptoms within days. Symptoms were brought under control following re-initiation of anakinra, demonstrating the requirement of daily maintenance with this drug (Goldbach-Mansky et al 2006; Hawkins et al 2006; Maksimovic et al 2008).

\section{ACZ885}

ACZ885 (Canakinumab), a fully humanized monoclonal antibody against IL-1 $\beta$, currently in phase III development, has demonstrated promising results during testing of the safety and pharmacokinetics for use of this therapy for treatment of CAPS (Kuemmerle-Deschner et al 2008; Lachmann et al 2006) and possibly other complex inflammatory diseases, such as RA (Alten et al 2008), systemic juvenile idiopathic arthritis (sJA), psoriasis, and inflammatory bowel disease. Monoclonal antibody therapies are often advantageous over other forms of biologic therapies due to their relative longer half-lives, thus ensuring full neutralization of the target over a longer period of time. In April 2008, phase II data were reported at the Fifth International Congress on Familial Mediterranean Fevers and Systemic Autoinflammatory Diseases in Rome, Italy. ACZ885 demonstrated long-lasting clinical remission in patients with CAPS. The 50-patient study treated adult CAPS patients with a $150 \mathrm{mg}$ injection of the drug every 8 weeks and pediatric patients with $2 \mathrm{mg} / \mathrm{kg}$. Patients' symptoms displayed improvement within 1 day and all achieved complete clinical remission within 7 days. The drug was well tolerated and patients remained in remission for an average of 115 days. Phase III studies are still ongoing (Lachmann et al 2008), but ACZ885 has recently been granted EU and US Orphan drug status for treatment of CAPS.

\section{Rilonacept (IL- I Trap/Arcalyst ${ }^{\mathrm{TM}}$ )}

In February 2008, Regeneron pharmaceuticals received 'Orphan Drug' approval from the Food and Drug Administration
(FDA) for rilonacept (IL-1 Trap/Arcalyst ${ }^{\mathrm{TM}}$ ) for the treatment of two CAPS disorders, FCAS and MWS, in adults and children aged 12 years and older. This made rilonacept the first therapy to be approved for the treatment of CAPS (Ratner 2008). Rilonacept is a fusion protein, the product of 'Trap' technology, designed to bind to and neutralize IL-1 before it can bind to cell surface receptors and induce inflammation. It is a dimeric glycoprotein, consisting of the human IL-1 receptor extracellular domains and the Fc portion of human IgG1, with a molecular weight of approximately $251 \mathrm{kDa}$. In a single molecule, rilonacept incorporates both receptor components required for signalling namely the IL-1 type 1 receptor and the IL-1 receptor accessory protein (AcP). One clear advantage of the structure of this fusion protein is the ability to bind to both IL- $1 \alpha$ and IL- $1 \beta$ with high affinity (Economides et al 2003). This has the potential to ensue that rilonacept better efficiency to inhibit IL-1 in vivo than other IL-1 targeted therapies. Further to this, rilonacept can be administered weekly by subcutaneous injection, as opposed to the daily administration required for anakinra.

The FDA based its approval upon phase III clinical studies supported by Regeneron Pharmaceuticals, Inc., which demonstrated the drugs safety and effectiveness (Hoffman et al 2008). Changes in disease activity were measured in 47 adult patients with CAPS (44 with FCAS and 3 with MWS), using a composite symptom score compiled from a daily questionnaire (Daily Health Assessment Form [DHAF]), evaluating signs of rash, feelings of fever/chills, joint pain, eye redness/pain, and fatigue, using a scale of zero (none/ no severity) to 10 (very severe) (Hoffman et al 2008). The study was conducted in a randomized, double-blind, placebo controlled manner in two parts (1 and 2$)$ in the same patients with FCAS and MWS. In study 1, patients either received $160 \mathrm{mg}$ weekly dose of rilonacept, after an initial loading dose of $320 \mathrm{mg}$, or placebo for 6 weeks. In Study 2, which followed immediately after study 1 , all subjects were given $160 \mathrm{mg}$ rilonacept weekly for 9 weeks in a patient-blind manner, followed by a 9 week randomized, double blind withdrawal period in which patients were randomly assigned to either remain on treatment at $160 \mathrm{mg}$ per week or to receive placebo. In study 1,24 patients received rilonacept, and a significant reduction in mean symptom score was demonstrated compared with the 23 patients that received the placebo for the same period. Furthermore, study 2, the mean symptom scores increased more in those patients withdrawn to placebo compared with patients who remained on rilonacept. Rilonacept was tolerated well by these patients. The most common adverse reactions reported were injection 
site reactions and upper respiratory tract infections, similar to those reported for anakinra treatment.

The design of this phase III study was based upon the findings of an initial pilot study of 5 patients with FCAS. In that open-label trial, a significant improvement, with sustained response and good tolerability to rilonacept, was reported (Goldbach-Mansky et al 2008). Following a baseline evaluation, all patients received an initial loading dose of $300 \mathrm{mg}$ of rilonacept by subcutaneous injection. The patients were then evaluated 6 and 10 days later for clinical efficacy, and remained off drug until a clinical flare occurred. At that time the patients received $300 \mathrm{mg}$ of rilonacept and were then maintained on $100 \mathrm{mg}$ weekly doses, as part of an extension study. Patients whose symptoms were not controlled (CRP $<0.5 \mathrm{mg} / \mathrm{dL}$ and/or SAA $<10 \mathrm{mg} / \mathrm{L}$ and a daily diary score of $<0.5$, not achieved) could have their dose increased to $160 \mathrm{mg}$ per week and then further to $320 \mathrm{mg}$ per week in an intra-patient dose escalation phase.

Compared to the phase III study, this pilot clinical trial assessed safety, disease activity (determined by 3 symptom [rash, joint pain/swelling, and fever] compiled score recorded in a daily diary), quality of health (SF-36 questionnaire) and also markers of inflammation (ESR, CRP, SAA and IL-6), at $3,6,9,12$ and 24 months post initiation of rilonacept administration, and compared with baseline measurements.

Within hours of receiving the initial dose of rilonacept, all patients reported benefit with a decrease in cold-induced attacks and improvements in symptoms as measured by reduction in daily scores. Three patients with hearing loss demonstrated no further deterioration during the two years of observation. Maximal clinical improvement was observed in four patients at day 10 , and one patient at day 6 . The improvement in daily scores was accompanied by decreases in ESR, CRP and SAA levels. All patients entered the extension phase, and completed 24 months of follow up at a dose of $160 \mathrm{mg} /$ week or higher. At the higher doses, a statistically significant improvement was seen in the reduction of the ESR $(\mathrm{p}<0.05)$, and a trend towards further improvement in CRP and SAA level reduction. Four of the five patients did not fulfil the criteria for CRP and the daily scores at $160 \mathrm{mg} /$ week dose and were subsequently increased to $320 \mathrm{mg} /$ week. The magnitude of improvement was less pronounced in the dose increase from $160 \mathrm{mg} /$ week to $320 \mathrm{mg}$ than that observed for $100 \mathrm{mg}$ up to $160 \mathrm{mg} /$ week. IL-6 levels were elevated at baseline but were decreased to undetectable levels at a dose of $160 \mathrm{mg} /$ week in all patients. These observations established $160 \mathrm{mg} /$ week as the favored dose for the subsequent phase III study described above.
The results of both of these studies are consistent, with rilonacept inducing a rapid and sustained reduction in the signs and symptoms of CAPS, regardless of the method of assessment. In the phase III study, rilonacept almost abolished the occurrence of flares, which was paralleled with decreases in the limitations of work, social and other activities. Although some patients showed the development of anti-rilonacept antibodies, there was no evidence that these inhibited the efficacy or safety of the drug. Whether there may be any further clinical significance of these antibodies is not yet determined and requires future follow-up. The patients tolerated rilonacept favorably in both studies, with reported adverse reactions considered only as mild or moderate.

Preliminary data suggests a beneficial effect of rilonacept in other inflammatory conditions including Familial Mediterranean Fever (FMF) (Hashkes et al 2008), sJIA (Lovell et al 2006a, b) and gouty arthritis (Radin et al 2008; Terkeltaub et al 2007).

\section{Conclusions}

The identification of the mutations in the CIAS1 gene and subsequent elucidation of the molecular mechanisms underlying the symptoms of CAPS accompanied with the development of IL-1 targeting biologics has been a triumph for translational research and a major therapeutic advance for patients suffering with CAPS. Indeed the future for CAPS patients has never seemed brighter, with current availability of not one but several biologic agents which will undoubtedly transform the lives of affected patients. For the medical community, the availability of anakinra, rilonacept and ACZ885 in their treatment armamentarium, has transformed the prognosis and treatment of patients from one of limited success to one of muted confidence, with the toughest decision being the choice of which biologic to prescribe. Ignoring the potential costs of each treatment, the ability to personalize treatment to the specific needs of individual patients is a powerful and welcome tool. The IL-1 blockade flagship, anakinra, has been pivotal in the determination of the molecular mechanisms of CAPS and a pioneer in the successful and sustained treatment of CAPS. Yet the frequent and high dose injections are not always tolerated well by patients. The desirable longer half-lives of rilonacept and ACZ885 offer potential alternatives to patients who tolerate daily injections poorly or have difficulty with drug compliance. The initial evidence for the beneficial effects of rilonacept for MWS and FCAS suggests that it might also be a suitable treatment for CINCA/NOMID, but as of yet no clinical trials of rilonacept have been performed in patients with 
this condition. It is yet to be determined whether rilonacept or ACZ885 will have any greater efficacy in treatment of other chronic inflammatory conditions, such as RA, where anakinra has up to now proven little benefit.

\section{Disclosures}

None of the authors have conflicts of interest to disclose.

\section{References}

Aganna E, Martinon F, Hawkins PN, et al. 2002. Association of mutations in the NALP3/CIAS1/PYPAF1 gene with a broad phenotype including recurrent fever, cold sensitivity, sensorineural deafness, and AA amyloidosis. Arthritis Rheum, 46:2445-52.

Agostini L, Martinon F, Burns K, et al. 2004. NALP3 forms an IL-1 betaProcessing inflammasome with increased activity in Muckle-Wells autoinflammatory disorder. Immunity, 20:319-25.

Aksentijevich I, Nowak M, Mallah M, et al. 2002. De novo CIAS1 mutations, cytokine activation, and evidence for genetic heterogeneity in patients with neonatal-onset multisystem inflammatory disease (NOMID): a new member of the expanding family of pyrin-associated autoinflammatory diseases. Arthritis Rheum, 46:3340-8.

Alten R, Gram H, Joosten LA, et al. 2008. The human anti-IL-1 beta monoclonal antibody ACZ885 is effective in joint inflammation models in mice and in a proof-of-concept study in patients with rheumatoid arthritis. Arthritis Res Ther, 10:R67.

Cassel SL, Eisenbarth SC, Iyer SS, et al. 2008. The Nalp3 inflammasome is essential for the development of silicosis. Proc Natl Acad Sci USA, 105:9035-40.

Church LD, Cook GP, McDermott MF. 2008. Primer: inflammasomes and interleukin 1 beta in inflammatory disorders. Nat Clin Pract Rheumatol, 4:34-42.

Clark W, Jobanputra P, Barton P, et al. 2004. The clinical and costeffectiveness of anakinra for the treatment of rheumatoid arthritis in adults: a systematic review and economic analysis. Health Technol Assess, 8:iii-x, 1.

Cuisset L, Drenth JP, Berthelot JM, et al. 1999. Genetic linkage of the Muckle-Wells syndrome to chromosome 1q44. Am J Hum Genet, 65:1054-9.

Dode C, Cuisset L, Delpech M, et al. 2003. TNFRSF1A-associated periodic syndrome (TRAPS), Muckle-Wells syndrome (MWS) and renal amyloidosis. J Nephrol, 16:435-7.

Dode C, Hazenberg BP, Pecheux C, et al. 2002a, Mutational spectrum in the MEFV and TNFRSF1A genes in patients suffering from AA amyloidosis and recurrent inflammatory attacks. Nephrol Dial Transplant, $17: 1212-7$.

Dode C, Le Du N, Cuisset L, et al. 2002b. New mutations of CIAS1 that are responsible for Muckle-Wells syndrome and familial cold urticaria: a novel mutation underlies both syndromes. Am J Hum Genet, 70:1498-506.

Dollfus H, Hafner R, Hofmann HM, et al. 2000. Chronic infantile neurological cutaneous and articular/neonatal onset multisystem inflammatory disease syndrome: ocular manifestations in a recently recognized chronic inflammatory disease of childhood. Arch Ophthalmol, 118:1386-92.

Economides AN, Carpenter LR, Rudge JS, et al. 2003. Cytokine traps: multicomponent, high-affinity blockers of cytokine action. Nat Med, 9:47-52.

Eisenbarth SC, Colegio OR, O'Connor W, et al. 2008. Crucial role for the Nalp3 inflammasome in the immunostimulatory properties of aluminium adjuvants. Nature, 453:1122-6.

Farasat S, Aksentijevich I, Toro JR. 2008, Autoinflammatory diseases: clinical and genetic advances. Arch Dermatol, 144:392-402.

Feldmann J, Prieur AM, Quartier P, et al. 2002. Chronic infantile neurological cutaneous and articular syndrome is caused by mutations in CIAS1, a gene highly expressed in polymorphonuclear cells and chondrocytes. Am J Hum Genet, 71:198-203.
Goldbach-Mansky R, Dailey NJ, Canna SW, et al. 2006. Neonatal-onset multisystem inflammatory disease responsive to interleukin-1 beta inhibition. N Engl J Med, 355:581-92.

Goldbach-Mansky R, Shroff SD, Wilson M, et al. 2008. A pilot study to evaluate the safety and efficacy of the long-acting interleukin-1 inhibitor rilonacept (interleukin-1 trap) in patients with familial cold autoinflammatory syndrome. Arthritis Rheum, 58:2432-42.

Haas N, Kuster W, Zuberbier T, et al. 2004. Muckle-Wells syndrome: clinical and histological skin findings compatible with cold air urticaria in a large kindred. Br J Dermatol, 151:99-104.

Hashkes P, Lovell D, Giannini E, et al. 2008. Rilonacept (IL-1 Trap) for treatment of colchicine resistant Familial Mediterranean Fever: A randomized, multicenter double-blinded, alternating treatment (N of 1) trial. Clin Exp Rheumatol, 26:193:N18.

Hashkes PJ, Lovell DJ. 1997. Recognition of infantile-onset multisystem inflammatory disease as a unique entity. J Pediatr, 130:513-5.

Hawkins PN, Bybee A, Aganna E, et al. 2004a. Response to anakinra in a de novo case of neonatal-onset multisystem inflammatory disease. Arthritis Rheum, 50:2708-9.

Hawkins PN, Lachmann HJ, Aganna E, et al. 2004b. Spectrum of clinical features in Muckle-Wells syndrome and response to anakinra. Arthritis Rheum, 50:607-12.

Hawkins PN, Lachmann HJ, McDermott MF. 2003. Interleukin-1receptor antagonist in the Muckle-Wells syndrome. $N$ Engl $J$ Med, $348: 2583-4$

Hoffman HM, Mueller JL, Broide DH, et al. 2001. Mutation of a new gene encoding a putative pyrin-like protein causes familial cold autoinflammatory syndrome and Muckle-Wells syndrome. Nat Genet, 29:301-5.

Hoffman HM, Rosengren S, Boyle DL, et al. 2004. Prevention of coldassociated acute inflammation in familial cold autoinflammatory syndrome by interleukin-1 receptor antagonist. Lancet, 364:1779-85.

Hoffman HM, Throne ML, Amar NJ, et al. 2008. Efficacy and safety of rilonacept (interleukin-1 trap) in patients with cryopyrin-associated periodic syndromes: Results from two sequential placebo-controlled studies. Arthritis Rheum, 58:2443-52.

Hoffman HM, Wright FA, Broide DH, et al. 2000. Identification of a locus on chromosome 1q44 for familial cold urticaria. Am J Hum Genet, 66:1693-8.

Kaufman RA, Lovell DJ. 1986. Infantile-onset multisystem inflammatory disease: radiologic findings. Radiology, 160:741-6.

Kile RL, Rusk HA. 1940. A case of cold urticaria with an unusual family history. JAMA, 114:1067-8.

Kuemmerle-Deschner JB, Tzaribachev N, Hausmann S, et al. 2008. Longlasting response to ACZ885 (a new human IgG1 anti-IL-1 beta monoclonal antibody) in patients with Muckle-Wells Syndrome (MWS). Clin Exp Rheumatol, 26:180:G2.

Lachmann H, Jung T, Felix S, et al. 2008. Treatment of cryopyrin associated periodic fever syndrome with a fully human anti-IL-1beta monoclonal antibody (ACZ885): results from a subcutaneous administration study. Clin Exp Rheumatol, 26:181:G3.

Lachmann HJ, Jung T, Bobadilla M, et al. 2006. Treatment of Muckle Wells syndrome with a fully human anti-IL-1beta monoclonal antibody (ACZ885) - Initial results from a proof of concept study. Ann Rheum Dis, 65(Suppl II):76.

Leslie KS, Lachmann HJ, Bruning E, et al. 2006. Phenotype, genotype, and sustained response to anakinra in 22 patients with autoinflammatory disease associated with CIAS-1/NALP3 mutations. Arch Dermatol, $142: 1591-7$

Lorber J. 1973. Syndrome for diagnosis: dwarfing, persistently open fontanelle; recurrent meningitis; recurrent subdural effusions with temporary alternate-sided hemiplegia; high-tone deafness; visual defect with pseudopapilloedema; slowing intellectual development; recurrent acute polyarthritis; erythema marginatum, splenomegaly and iron-resistant hypochromic anaemia. Proc R Soc Med, 66:1070-1.

Lovell D, Giannini E, Kimura Y, et al. 2006a. Preliminary evidence for bioactivity of IL-1 trap (rilonacept), a long acting IL-1 inhibitor, in systemic juvenile idiopathic arthritis. Ann Rheum Dis, 65(Suppl II):693. 
Lovell DJ, Giannini EH, Kimura Y, et al. 2006b. Preliminary evidence for bioactivity of IL-1 trap (Rilonacept), a long acting IL-1 inhibitor, in systemic juvenile idiopathic arthritis (SJIA). Arthritis Rheum, 54:S325-S6.

Maksimovic L, Stirnemann J, Caux F, et al. 2008. New CIAS1 mutation and anakinra efficacy in overlapping of Muckle-Wells and familial cold autoinflammatory syndromes. Rheumatology (Oxford), 47:309-10.

Martinon F, Burns K, Tschopp J. 2002. The inflammasome: a molecular platform triggering activation of inflammatory caspases and processing of proIL-beta. Mol Cell, 10:417-26.

Martinon F, Petrilli V, Mayor A, et al. 2006. Gout-associated uric acid crystals activate the NALP3 inflammasome. Nature, 440:237-41.

McDermott MF, Aksentijevich I. 2002. The autoinflammatory syndromes. Curr Opin Allergy Clin Immunol, 2:511-6.

Mirault T, Launay D, Cuisset L, et al. 2006. Recovery from deafness in a patient with Muckle-Wells syndrome treated with anakinra. Arthritis Rheum, 54:1697-700.

MuckleT, Wells M. 1962. Urticaria, deafness, and amyloidosis: a new heredo-familial syndrome. $Q J \mathrm{Med}, 31: 235-48$.

Muruve DA, Petrilli V, Zaiss AK, et al. 2008. The inflammasome recognizes cytosolic microbial and host DNA and triggers an innate immune response. Nature, 452:103-7.

Neven B, Prieur AM, Dit Maire PQ. 2008. Cryopyrinopathies: update on pathogenesis and treatment. Nat Clin Pract Rheumatol, 4:481-9.

O'Connell SM, O'Regan GM, Bolger T, et al. 2007. Response to IL-1receptor antagonist in a child with familial cold autoinflammatory syndrome. Pediatr Dermatol, 24:85-9.

Petrilli V, Papin S, Dostert C, et al. 2007. Activation of the NALP3 inflammasome is triggered by low intracellular potassium concentration. Cell Death Differ, 14:1583-9.
Prieur AM, Griscelli C, Lampert F, et al. 1987. A chronic, infantile, neurological, cutaneous and articular (CINCA) syndrome. A specific entity analysed in 30 patients. Scand J Rheumatol Suppl, 66:57-68

Radin A, Terkeltaub R, Schumacher H, et al. 2008. Placebo-controlled pilot study of rilonacept (IL-1 Trap), a long acting IL-1 inhibitor, in refractory chronic active gouty arthritis. Clin Exp Rheum, 26:181:G5.

Ratner M. 2008. IL-1 trap go-ahead. Nat Biotechnol, 26:485.

Rosengren S, Mueller JL, Anderson JP, et al. 2007. Monocytes from familial cold autoinflammatory syndrome patients are activated by mild hypothermia. J Allergy Clin Immunol, 119:991-6.

Ross JB, Finlayson LA, Klotz PJ, et al. 2008. Use of anakinra (Kineret) in the treatment of familial cold autoinflammatory syndrome with a 16-month follow-up. J Cutan Med Surg, 12:8-16.

Rynne M, Maclean C, Bybee A, et al. 2006, Hearing improvement in a patient with variant Muckle-Wells syndrome in response to interleukin 1 receptor antagonism. Ann Rheum Dis, 65:533-4.

Taylor PC. 2003. Anti-cytokines and cytokines in the treatment of rheumatoid arthritis. Curr Pharm Des, 9:1095-106.

Terkeltaub R, Schumacher HR, Sundy J, et al. 2007. Placebo-controlled pilot study of rilonacept (IL-1 trap), a long acting IL-1 inhibitor, in refractory chronic active gouty arthritis. Arthritis Rheum, 56:4231-2.

Ting JP, Kastner DL, Hoffman HM. 2006. CATERPILLERs, pyrin and hereditary immunological disorders. Nat Rev Immunol, 6:183-95.

Torbiak RP, Dent PB, Cockshott WP. 1989. NOMID - a neonatal syndrome of multisystem inflammation. Skeletal Radiol, 18:359-64.

Touitou I, Savic S, Mathews R, et al. 2008. Fifth International Congress on Familial Mediterranean Fever and Systemic Autoinflammatory Diseases. Exp Rev Clin Immunol, 4:425-8. 